\title{
Universal Effect of Excitation Dispersion on the Heat Capacity and Gapped States in Fluids
}

\author{
Nikita P. Kryuchkov, ${ }^{1}$ Lukiya A. Mistryukova, ${ }^{1}$ Andrei V. Sapelkin, ${ }^{2}$ Vadim V. Brazhkin, ${ }^{3}$ Stanislav O. Yurchenko ${ }^{1}$ * \\ ${ }^{1}$ Bauman Moscow State Technical University, 2nd Baumanskaya str. 5, 105005 Moscow, Russia \\ ${ }^{2}$ School of Physics and Astronomy, Queen Mary University of London, London E1 4NS, England \\ ${ }^{3}$ Institute for High Pressure Physics RAS, Kaluzhskoe shosse, 14, Troitsk, Moscow, 108840 Russia
}

(Dated: September 17, 2020)

\begin{abstract}
The change in dispersion of high-frequency excitations in fluids, from oscillating solid-like to monotonous gas-like one, is shown for the first time to affect thermal behavior of heat capacity and the $q$-gap width in reciprocal space. With in silico study of liquified nobel gases, liquid iron, liquid mercury, and model fluids, we established universal bilinear dependence of heat capacity on $q$-gap width, whereas the crossover precisely corresponds to the change in the excitation spectra. The results open novel prospects for studies of various fluids, from simple to molecular liquids and melts.
\end{abstract}

Understanding physical properties of condensed matter for given interatomic interactions and thermodynamic conditions is a long-standing problem in materials science with the most impressive progress achieved in crystals 1, 2. Success in the lattice dynamics theory of crystals elicited attempts to generalize crystalline approaches to non-periodic systems, e.g., for glasses, where diffusion is extremely slow and atoms oscillate with small amplitudes around equilibrium positions [3]. However, this approach cannot be used in fluids, where neither assumptions of periodicity nor of small displacements hold.

Particle dynamics in fluids represents strongly coupled motion in an anharmonic dynamically-changing energy landscape. Displacements in liquids are large and the interactions are strong compared to the corresponding kinetic energy - condition known as the absence of small parameter. Enhanced anharmonicity leads to strong interaction between excitations, resulting in their intensive damping, generation, and spectra redistribution. As a consequence, understanding relationships between collective dynamics (i.e. excitation spectra) and thermodynamics (e.g. heat capacity) in fluids remains an outstanding and challenging problem.

Experimentally, excitation spectra in fluids can be obtained with inelastic neutron and x-ray scattering [9 17]. Yet another approach is to use complex plasmas - the model systems of charged and strongly-interacting particles levitating in a weakly-ionized gas, whose interactions can be tuned, and the motions can be visualized [18]21]. Molecular dynamics (MD) simulations are also widely used [17, 22 34 to study collective dynamics in systems with a range of interatomic interactions and, crucially, allow access to a broad range of parameters, including the ones that may not be accessible experimentally. For instance, embedded atom model (EAM) potentials can be used to study heat capacity [35] 37] and excitation spectra 29, 31] (for detailed analysis of EAM potentials used for liquid metals, see [36]). Thus, detailed analysis of MD data allows to obtain dispersion curves and lifetimes of excitations for all wavenumbers $q$ [32].

A well-known general feature of transverse excitation spectra in fluids is existence of the gap in the reciprocal space - $q$-gap - associated with instability of the long wavelength transverse excitations [32, 38,41]. The value of $q$-gap (characterizing transverse excitations at small q) has been assumed [30, 41, 42, to influence the heat capacity of fluids, but this has never been tested directly in either experiments or MD simulations.

Another generic phenomenon recently studied with MD simulations, theoretically, and experimentally with the model fluids [21, 43] is anticrossing of longitudinal and transverse excitations. It has been demonstrated that structural disorder and ahnarmonicity induce mutual mixing of the modes at large $q$. As result, the modes repel, forming a frequency gap, accompanied by the strong redistribution and hybridization of the spectra in the region of mode crossing. It was also found that with temperature increase, the dispersion of high-frequency excitations (corresponding to longitudinal waves at small $q$ ) changes from oscillating (inherent to solids) to monotoniously-growing shape (inherent to gasses). However, the effect of this change on thermodynamics of fluids has not been studied as yet.

In this article, we investigated for the first time dependence of isochoric heat capacity on the $q$-gap width for a range of simple fluids and reveal a clear evidence of the change from solid- to gas-like dispersion of highfrequency (longitudinal at small $q$ ) excitations affecting thermodynamics of fluids. By doing so we discover a universal relationship between the excitation spectra dispersion, the width of $q$-gap, and the heat capacity of fluids.

Details of MD simulations. - The simulations of fluids were performed in a wide range of temperatures and densities. We considered Lennard-Jones (LJ) potential corresponding to noble gasses 44 46, inverse-power-law repulsion $\propto 1 / r^{k}$ (IPLk, we considered IPL8 and IPL12 fluids) 47, 48, and embedded atomic model (EAM) of liquid iron [49] and liquid mercury [37]:

$$
\frac{\varphi_{\mathrm{LJ}}(r)}{\epsilon}=4\left[\left(\frac{\sigma}{r}\right)^{12}-\left(\frac{\sigma}{r}\right)^{6}\right], \frac{\varphi_{\mathrm{IPL}}(r)}{\epsilon}=\left(\frac{\sigma}{r}\right)^{k},
$$

where $r$ is the distance between a pair of particles, $\epsilon$ 

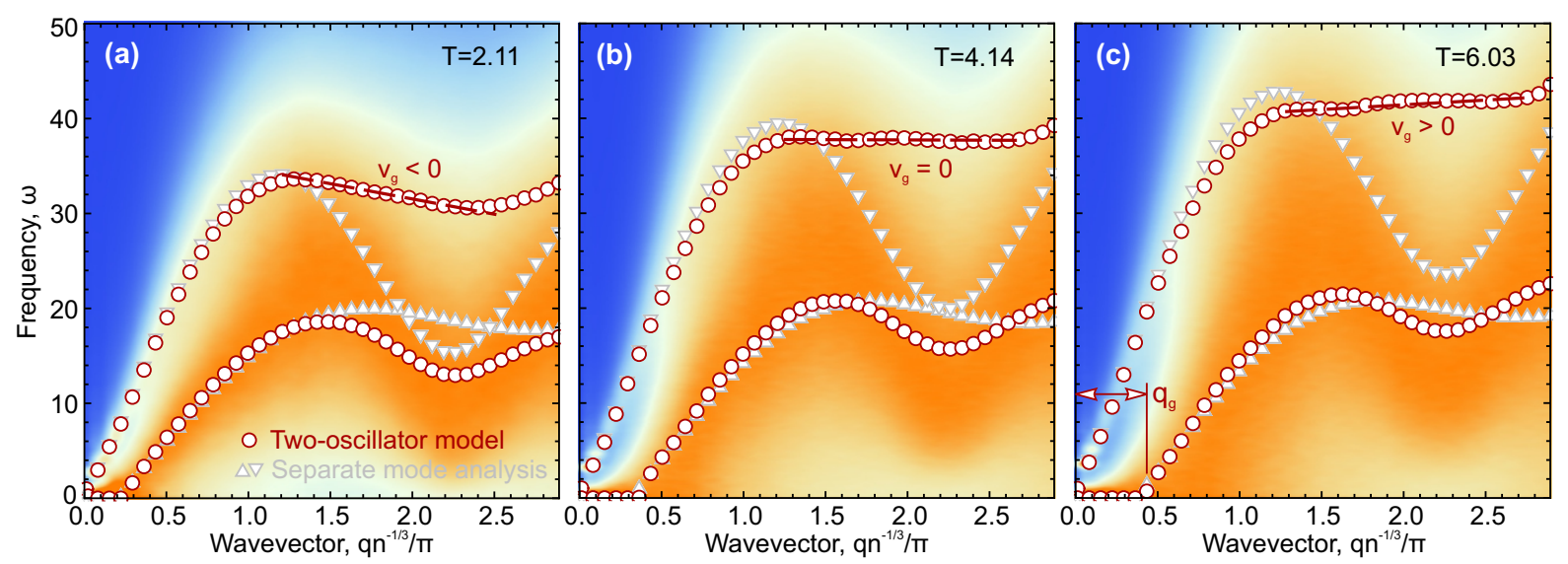

Figure 1. The change of spectra with temperature increase: The excitation spectra in $3 \mathrm{D}$ LJ fluid at $n=1$ are shown in (a-c) at the temperatures $T=2.11, T=4.14$, and $T=6.03$, respectively. The dashed red lines in (a-c) correspond to constant negative, zero, and positive group velocity $v_{g}$ of high-frequency excitations, illustrating the transition from solid- to gas-like dispersion. The region of $q$-gap is illustrated in (c). The colormap is $C(q, \omega)$ normalized to the maxima at each $q$, red circles and grey triangles are low- and high-frequency (hybridized) modes and test modes (separately analyzed), respectively.

and $\sigma$ are the energy and length scales of interaction, respectively, whereas, in the EAM model, the energy of $i$-th particle is:

$$
E_{i}=F\left(\rho_{i}\right)+\frac{1}{2} \sum_{j \neq i} \varphi_{p}\left(r_{i j}\right), \quad \rho_{i}=\sum_{j \neq i} \rho\left(r_{i j}\right),
$$

where $F\left(\rho_{i}\right)$ is the embedding energy determined by the local electron density $\rho_{i}$ arising from the contributions $\rho\left(r_{i j}\right)$ provided by other $j$-th particles, $\varphi_{p}\left(r_{i j}\right)$ is the pairwise interaction between $i$-th and $j$-th particles with the relative distance $r_{i j}$, and the summation is over all $j$-th particles. We used the functions $F(\rho), \rho(r)$, and $\varphi_{p}(r)$ reported for liquid iron in Ref. [49]. We studied liquid Fe at the densities $\rho=8$ and $10 \mathrm{~g} / \mathrm{cm}^{3}$ (corresponding to the Moon and Earth outer cores [50, 51]), and liquid Hg at $\rho=13.546 \mathrm{~g} / \mathrm{cm}^{3}$ (normal conditions).

We used 3D fluids consisting of $N=10^{4}$ particles in NVT ensemble with Nose-Hoover thermostat and at constant volume. The interaction cut-off radius was chosen as $r_{c}=5.0 n^{-1 / 3}$ (here $n=N / V$ is the particle density). For LJ and IPLk system, we chose numerical time step as $\Delta t=5 \times 10^{-3} \sqrt{T_{0} m \sigma^{2} /(T \epsilon)}$ and $T_{0} / \epsilon=0.01$; we used $\Delta t=0.1 \sqrt{T_{0} / T}$ fs and $T_{0}=7 \times 10^{3} \mathrm{~K}$ for liquid iron and $\Delta t=0.075 \sqrt{T_{0} / T}$ fs and $T_{0}=600 \mathrm{~K}$ for liquid mercury. Simulations were performed in dimensionless units $(\epsilon=1, \sigma=1$, and $m=1)$ for LJ and IPLk systems and in SI units for EAM systems. To make sure that the results are technically correct, we analyzed systems with enlarged size, half timestep, and NVE ensemble (see SM for details [52]). All simulations were run for $2 \times 10^{6}$ time steps in LAMMPS package [53]. The first $5 \times 10^{5}$ steps were used for system equilibration, and the following steps we used for calculation of properties.

The excitation spectra were calculated with the methods developed in Refs. [32, 43. At the first step, we calculated particle current spectra $C_{L, T}(\mathbf{q}, \omega)$ [38]:

$$
C_{L, T}(\mathbf{q}, \omega)=\int d t e^{i \omega t} \operatorname{Re}\left\langle\mathbf{j}_{L, T}(\mathbf{q}, t) \mathbf{j}_{L, T}(-\mathbf{q}, 0)\right\rangle,
$$

where $\mathbf{j}(\mathbf{q}, t)=N^{-1} \sum_{s} \mathbf{v}_{s}(t) \exp \left(i \mathbf{q} \mathbf{r}_{s}(t)\right)$ is the velocity current; $\mathbf{v}_{s}(t)=\dot{\mathbf{r}}_{s}(t)$ is the velocity of the $s$-th particle; $\mathbf{q}$ is wavevector; $\mathbf{j}_{L}=\mathbf{q}(\mathbf{j} \cdot \mathbf{q}) / q^{2}$ and $\mathbf{j}_{T}=\mathbf{j} \cdot \mathbf{e}_{\perp}$ are the longitudinal and transverse components of the current, $\mathbf{e}_{\perp}$ is a unit vector normal to $\mathbf{q}$; the summation is performed over all $N$ particles in the system; the brackets $\langle\ldots\rangle$ denote the canonical ensemble average. Because of simple fluids are isotropic, the spectra depend only on $q=|\mathbf{q}|$ and we used this to enhance the accuracy of $C_{L, T}(q, \omega)$ calculation. Then, $C_{L, T}(q, \omega)$ data were analyzed with the separate mode analysis and the two-oscillator model [32, 43, to obtain dispersion curves $\omega_{l, h}(q)$ (for low- and high-frequency branches) and $q$-gap width. Using the two-oscillator model, the frequencies and damping rates of excitations are obtained by fitting the total current $C(q, \omega)=C_{L}(q, \omega)+2 C_{T}(q, \omega)$ by the sums of Lorentzians attributed to the high- and the lowfrequency oscillators [32, 43, (see details in Supplementary Materials (SM) [52]). The two oscillator model and mode anticrossing, in particular, played the key role in our approach to the analysis of excitation spectra.

Results. - The excitation spectra of LJ fluid at the normalized density $n=1$ calculated at different temperatures are shown in Fig. 1. Here, the total particle current spectra $C(q, \omega)$ are shown in a color-coded format (normalized to the maxima at each $q$ ), the grey triangles correspond to dispersion curves obtained with the commonly used separate mode analysis, whereas the red circles are high- and low-frequency hybridized modes calculated with two-oscillator model [32,52. Both approaches give practically the same results in hydrodynamic limit, 


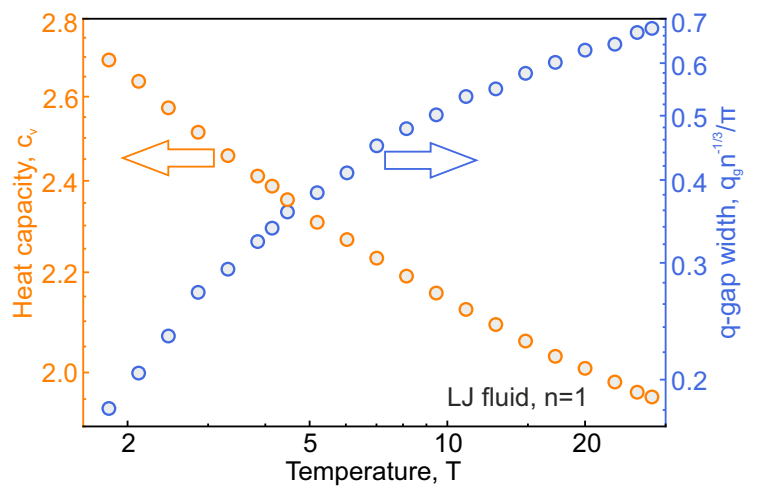

Figure 2. The temperature dependencies of heat capacity $c_{V}$ (orange symbols) and the width of $q$-gap $q_{g}$ (blue symbols) in LJ fluid at $n=1$. Similar dependencies for liquid Fe, IPL8, and IPL12 fluids are shown in Fig. S2 in SM [52.

$q n^{-1 / 3} / \pi \lesssim 1$ (see Fig. S1 in SM [52]). However, the difference becomes apparent at $q n^{-1 / 3} / \pi>1$ corresponding to the region of mode anticrossing [21, 43] and is a consequence of formation of hybridized low- and high- frequency modes (red circles) instead of the crossed dispersion curves (grey triangles). The high-frequency mode, associated with longitudinal (sound) excitations at small $q$, behaves as $\omega_{h}(q) \propto \sqrt{T} q$ at large $q[9,32,43,54$, and, beginning from some temperature, the high-frequency dispersion curve transforms from oscillating form (with sign-changing group velocity $v_{g}=\partial \omega_{h} / \partial q$, inherent for solids) to gas-like dispersion with $v_{g}>0$ at all $q$. Simultaneously, the separate modes (triangles in Fig. 1) are no longer crossing, but the hybridized modes (circles in Fig. (1) still repulse due to overlapping of $C_{L, T}$ caused by the mode damping (see in Figs. 1 (b), (c)).

The spectral ranges of the solid-like, intermediate, and gas-like of the high-frequency branch, shown in Figs. 1(a)-(c) at low, moderate, and high temperatures, as well as temperature dependencies $c_{V}(T)$ and $q_{g}(T)$, shown in Fig. 2, are specific for all fluids we studied. The temperature dependencies of the isochoric heat capacity, $c_{V}$, and the width of $q$-gap, $q_{g}$ (obtained from the excitation spectra in Fig. 1), for LJ fluid are shown in Fig. 2 by orange and blue symbols, respectively. These functions are nonlinear, smooth, and behave monotonously, including the region in vicinity of the temperature $T=4.14$, at which a broad region with $v_{g}=0$ appears in Fig. 1(b), and show no notable features. In a manner typical for fluids, the heat capacity $c_{V}$ drops from $c_{V} \simeq 3$ in vicinity of freezing line to the gaseous value $3 / 2$ at high temperatures [32, 35, 41].

It is then surprising that dependencies of heat capacity on the $q$-gap width $\left(c_{V}\left(q_{g}\right)\right.$, shown in Fig. 3(a)) display a clear bilinear behaviour and can be described by two linear functions with crossover points common for LJ, IPLs and EAM Fe systems $\left(q_{g} n^{-1 / 3} / \pi \simeq 0.34\right)$ and slightly different for mercury $\left(q_{g} n^{-1 / 3} / \pi \simeq 0.4\right)$. The
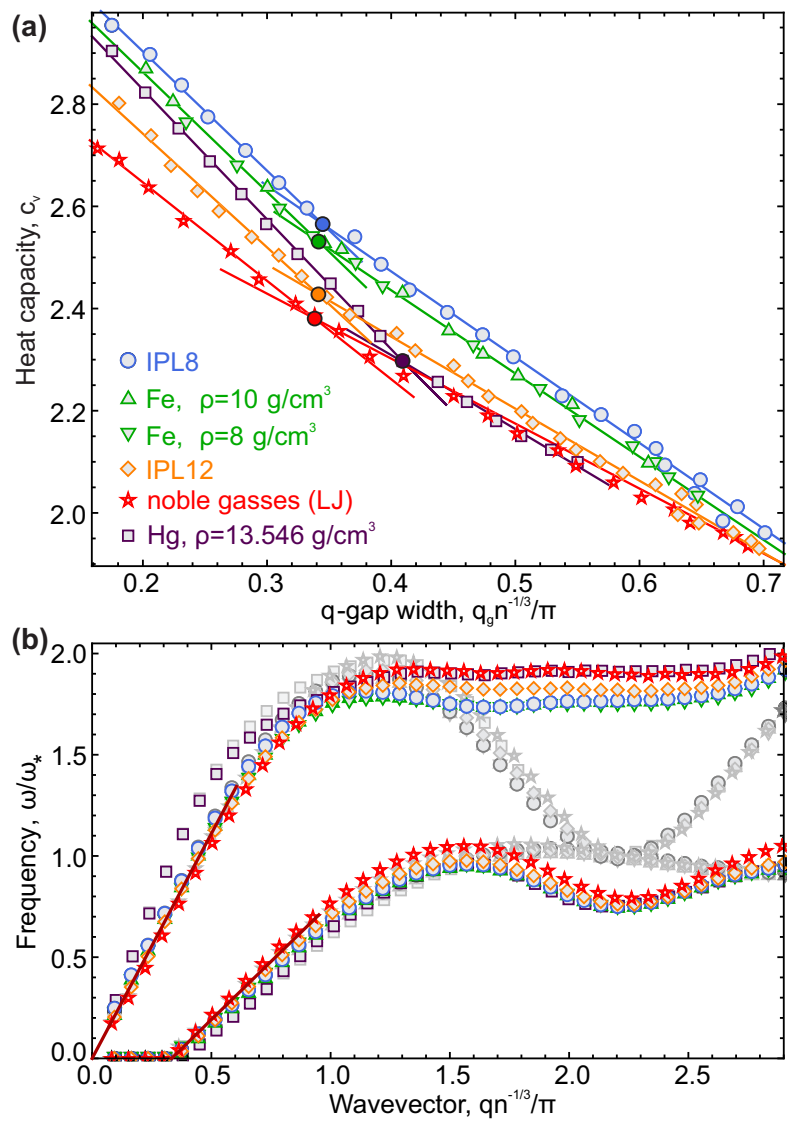

Figure 3. The relation between heat capacity and excitation spectra in the fluids under consideration: (a) The dependencies $c_{V}\left(q_{g}\right)$ in IPL8, IPL12, LJ fluids, liquid $\mathrm{Fe}$ and $\mathrm{Hg}$. Symbols correspond to MD results, solid lines are linear approximations with the kinks shown by the filled symbols. (b) Dispersion curves in the states corresponding to the kink points in (a). The colored and grey symbols were obtained with two-oscillator model (hybridyzed anticrossed modes) and separate-mode analysis (test modes), respectively [32. All frequencies in (b) are normalized to $\omega_{*}$ corresponding to the frequency of the test modes' touching. The red lines are linear fits for acoustic branch of dispersion curves and in vicinity of the $q$-gap boundary.

latter is caused by the interaction softness: LJ, IPL, and EAM Fe systems share a similar type of pairwise repulsion, whereas EAM Hg potential is softer and described with a set of polynomials. The functions $c_{V}(T)$ are smooth (with high accuracy) and, thus, the observed bilinear dependence can only originate from the change in the growth rate of $q_{g}(T)$ with temperature in each of the fluids. This result cannot be obtained with simplified crystalline-based approaches [30, 41, 42. Below, we show that this universal behavior is directly related to the changes of the excitations spectra shown in Fig. 1 .

In order to understand the origins of the observed universal behaviour, we referred back to the original excitation spectra for each of the fluids. We found a common feature in all cases: high-frequency dispersion 
curves $\omega_{h}(q)$ attain a broad plateau with $v_{g} \simeq 0$ at $q n^{-1 / 3} / \pi \simeq(1.5 \ldots 2.5)$, whereas dispersion curves $\omega_{L}(q)$ and $\omega_{T}(q)$ calculated by separate mode analysis just touch each other, as shown by grey symbols in Fig. 3(b). Here, the frequencies are normalized to the frequency of the grey curves touching $\left(\omega_{*}\right)$ and also exhibit very similar behavior in all fluids studied here. We found that the change in the shape of the excitation spectra from solidto gas-like on (and disappearance of the intersection of separate modes) correlates perfectly with the change in $q_{g}$ temperature behavior. Overall, this analysis provides clear evidence of the correlation between the change in excitation dispersion and behaviour of the heat capacity in fluids.

The crossing point shown in Fig. 3(a) for noble gasses (LJ fluid) corresponds to $\mathrm{Ne}$ at density $1.55 \mathrm{~g} / \mathrm{cm}^{3}$ and temperature $155 \mathrm{~K}$; Ar at $1.54 \mathrm{~g} / \mathrm{cm}^{3}$ and $486 \mathrm{~K}$; $\mathrm{Kr}$ at $2.48 \mathrm{~g} / \mathrm{cm}^{3}$ and $682 \mathrm{~K} 45$. In liquid $\mathrm{Fe}$, the change in slopes corresponds to approximately $4000 \mathrm{~K}$ at $8 \mathrm{~g} / \mathrm{cm}^{3}$ and $8000 \mathrm{~K}$ at $10 \mathrm{~g} / \mathrm{cm}^{3}$, whereas in liquid $\mathrm{Hg}$ it is at $1100 \mathrm{~K}$. The crossing points are located in the temperature range $T \simeq(2.3 \ldots 2.9) T_{m}$, from IPL8 to LJ fluids (here, $T_{m}$ is the melting temperature in the corresponding crystal), and at $T \simeq 4.7 T_{m}$ for liquid mercury (due to very soft short-range repulsion). At the same time, the offset between their $c_{V}$ plots vanishes with the $q$-gap width increase at higher temperatures, where all the systems exhibit similar gas-like behavior, as expected.

The data shown in Fig. 3(a) indicate that the interaction softness plays a key role in the observed $c_{V}\left(q_{g}\right)$ behavior: the softer an interaction is, the higher is the corresponding rate of decrease in the heat capacity, $\partial c_{V}\left(q_{g}\right) / \partial q_{g}$, before the crossing point. To analyse the role of repulsion range, attraction and many-body interactions, consider IPL8, liquid iron, IPL12, and LJ (noble gas) fluid. The systems with harder interactions typically have lower values of heat capacity near the melting temperature [55]. The IPL8 fluid has the strongest long-ranged repulsion, thus, the effects of anharmonicity are less pronounced here, compared to the other cases. Behavior, very similar to the IPL8 fluid, corresponds to the cases of $\mathrm{Fe}$, because of the pair part of the interaction $\varphi_{p}(r) \propto r^{-8.137}$ [49] is close to IPL8 and much softer than IPL12. At the same time, the many-body effects are not found to contribute significantly, as justified by the agreement of the results at the densities 8 and $10 \mathrm{~g} / \mathrm{cm}^{3}$. Comparing IPL12 and LJ fluids, we see that the attractive branch of interaction makes LJ fluid to behave more hard-sphere-like (the same conclusion was made during analysis of IPL12 and LJ crystals [56]). Liquid $\mathrm{Hg}$ exhibits the same characteristics, having the largest decrease rate $\partial c_{V}\left(q_{g}\right) / \partial q_{g}$ before the crossing point, LJ-like behavior after that, and enlarged sound velocity due to softness and long range of interactions, as we see in Fig. 3(b). We note, that the case of liquid $\mathrm{Hg}$ lends itself well for future experiments, since it does not require particularly challenging experimental conditions.

Discussion and Conclusions. - With MD simulations and methods for excitation analysis we recently developed [32, 43, we studied liquified noble gases, liquid iron, liquid mercury, and model fluids of particles interacting via inverse-power-law potentials, to understand the roles of repulsion softness, attractive and many-body interactions in dynamics and thermodynamics. Remarkably, we found that the heat capacity $c_{V}$ depends linearly on the $q$-gap width in a broad range of temperatures, but has a clear bilinear behaviour with a common crossover point, whereas the functions $c_{V}$ and $q_{g}$ themselves are smooth and essentially nonlinear in temperature. In all the studied fluids, the crossover position precisely correlates with the change in dispersion of high-frequency excitation spectra, from oscillating solid- to monotonouslygrowing gas-like one.

The change in slope of $c_{V}\left(q_{g}\right)$ in Fig. 3(a) corresponds exactly to the temperature when the top branch of the spectra in Fig. 1 develops the gas-like character $\left(v_{g}(q) \simeq 0\right.$ in a broad range of $\left.q\right)$. Since the group velocity is responsible for transport phenomena, we expect that plethora of unusual behavior in diffusion, viscosity, and thermal conductivity in fluids should be related to the observed change in spectra we revealed. Therefore, we believe that our findings will have far reaching implications for interpretation of already existing experimental data.

In fact, observation of experimental dynamic anomalies has long and rich history. For example, in low frequency Raman scattering in liquid water and glycerol, where such effects have been observed [57 59], but have never been satisfactorily explained since the findings reported here have never been considered. More recently, the change from solid- to gas-like dispersion for highfrequency excitations we described is likely to be responsible for anomalies observed experimentally and in simulations in Refs. 60 62. There, liquid metals were reported to behave more solid-like on cooling starting at a temperature $T \simeq(1.3 \ldots 1.7) T_{m}$ in isobaric experiments (that agrees with the crossover temperatures we observed on isochores in LJ, IPL, and Fe systems). This was associated with the change in dynamic regime, playing a crucial role as a precursor to structural freezing and important for understanding similarities and differences between liquids and glasses. However, the exact nature of the observed anomalies and of the dynamic regime change has never been established until now. It is an exciting prospect that the findings reported here provide fresh opportunities to utilise these well-established techniques for investigation of dynamics of liquids.

Our results provide new insights into the relationships between thermodynamic properties and excitations in fluids, opening novel prospects for theoretical and experimental studies of dynamics, thermodynamics, and transport phenomena in fluids of diverse nature, from simple 
liquids and melts to ionic, molecular liquids and solutions. Both the heat capacity and excitation spectra can be measured in experiments with noble gases or liquid metals, but the corresponding systematic studies have not been performed yet. We believe that our work will stimulate experiments and discussions aimed to detailed understanding how thermodynamics and transport phenomena are related to collective dynamics in fluids and will provide a basis for the development of generalised theoretical treatment of the liquid state dynamics.

The study is supported by the Russian Science Foundation, Grant No. 17-19-01691. N.P.K., L.A.M., and S.O.Y. are grateful to BMSTU State Assignment for infrastructural support.

* st.yurchenko@mail.ru

[1] M. T. Dove, Introduction to Lattice Dynamics (Cambridge University Press, 1993).

[2] A. Maradudin, Theory of lattice dynamics in the harmonic approximation, Solid state physics: Supplement (Academic Press, 1963).

[3] T. S. Grigera, V. Martín-Mayor, G. Parisi, and P. Verrocchio, Journal of Physics: Condensed Matter 14, 2167 (2002)

[4] V. I. Clapa, T. Kottos, and F. W. Starr, The Journal of Chemical Physics 136, 144504 (2012)

[5] Y. M. Beltukov, V. I. Kozub, and D. A. Parshin, Physical Review B 87, 134203 (2013)

[6] M. Baggioli and A. Zaccone, Phys. Rev. Research 1, 012010 (2019)

[7] M. Baggioli, R. Milkus, and A. Zaccone, Physical Review E 100, 062131 (2019)

[8] A. Zaccone, Journal of Physics: Condensed Matter 32, $203001(2020)$

[9] T. Scopigno, U. Balucani, A. Cunsolo, C. Masciovecchio, G. Ruocco, F. Sette, and R. Verbeni, Europhysics Letters (EPL) 50, 189 (2000).

[10] T. Scopigno, U. Balucani, G. Ruocco, and F. Sette, Journal of Physics: Condensed Matter 12, 8009 (2000)

[11] T. Scopigno, U. Balucani, G. Ruocco, and F. Sette, Physical Review E 65, 031205 (2002)

[12] T. Scopigno, G. Ruocco, and F. Sette, Reviews of Modern Physics 77, 881 (2005)

[13] W.-C. Pilgrim and C. Morkel, Journal of Physics: Condensed Matter 18, R585 (2006)

[14] S. Hosokawa, W.-C. Pilgrim, H. Sinn, and E. E. Alp, Journal of Physics: Condensed Matter 20, 114107 (2008)

[15] S. Hosokawa, M. Inui, Y. Kajihara, K. Matsuda, T. Ichitsubo, W.-C. Pilgrim, H. Sinn, L. E. González, D. J. González, S. Tsutsui, and A. Q. R. Baron, Physical Review Letters 102, 105502 (2009)

[16] V. M. Giordano and G. Monaco, Proceedings of the National Academy of Sciences 107, 21985 (2010).

[17] S. Hosokawa, S. Munejiri, M. Inui, Y. Kajihara, W.-C. Pilgrim, Y. Ohmasa, S. Tsutsui, A. Q. R. Baron, F. Shimojo, and K. Hoshino, Journal of Physics: Condensed Matter 25, 112101 (2013)

[18] S. Nunomura, S. Zhdanov, D. Samsonov, and G. Morfill,
Physical Review Letters 94, 045001 (2005).

[19] V. Nosenko, J. Goree, and A. Piel, Physical Review Letters 97, 115001 (2006).

[20] N. P. Kryuchkov, E. V. Yakovlev, E. A. Gorbunov, L. Couedel, A. M. Lipaev, and S. O. Yurchenko, Physical Review Letters 121, 075003 (2018)

[21] E. V. Yakovlev, N. P. Kryuchkov, P. V. Ovcharov, A. V. Sapelkin, V. V. Brazhkin, and S. O. Yurchenko, The Journal of Physical Chemistry Letters 11, 1370 (2020)

[22] P. Schmidt, G. Zwicknagel, P. G. Reinhard, and C. Toepffer, Physical Review E 56, 7310 (1997).

[23] H. Ohta and S. Hamaguchi, Physical Review Letters 84, 6026 (2000).

[24] P. Hartmann, Z. Donko, G. J. Kalman, S. Kyrkos, M. Rosenberg, and P. M. Bakshi, IEEE Transactions on Plasma Science 35, 337 (2007)

25] K. I. Golden, G. J. Kalman, P. Hartmann, and Z. Donkó, Physical Review E 82, 036402 (2010)

[26] J. Goree, Z. Donkó, and P. Hartmann, Physical Review E 85, 066401 (2012)

[27] J.-F. Wax and T. Bryk, Journal of Physics: Condensed Matter 25, 325104 (2013).

[28] S. Khrapak, B. Klumov, and L. Couedel, Scientific Reports 7, 7985 (2017)

[29] R. M. Khusnutdinoff, B. N. Galimzyanov, and A. V. Mokshin, Journal of Experimental and Theoretical Physics 126, 83 (2018).

[30] Y. D. Fomin, V. N. Ryzhov, E. N. Tsiok, J. E. Proctor, C. Prescher, V. B. Prakapenka, K. Trachenko, and V. V. Brazhkin, Journal of Physics: Condensed Matter 30, 134003 (2018),

[31] A. V. Mokshin and B. N. Galimzyanov, Journal of Physics: Condensed Matter 30, 085102 (2018).

[32] N. P. Kryuchkov, L. A. Mistryukova, V. V. Brazhkin, and S. O. Yurchenko, Scientific Reports 9, 10483 (2019)

[33] S. A. Khrapak, A. G. Khrapak, N. P. Kryuchkov, and S. O. Yurchenko, The Journal of Chemical Physics 150, 104503 (2019)

[34] D. C. Wallace, S. Rudin, G. D. Lorenzi-Venneri, and T. Sjostrom, Physical Review B 99, 104204 (2019).

[35] M. Forsblom and G. Grimvall, Physical Review B 72, 132204 (2005)

[36] D. K. Belashchenko, Physics-Uspekhi 56, 1176 (2013).

[37] D. K. Belashchenko, High Temperature 51, 40 (2013).

[38] J.-P. Hansen and I. R. MacDonald, Theory of simple liquids (Academic, London, 2006).

[39] T. Bryk, F. Gorelli, G. Ruocco, M. Santoro, and T. Scopigno, Physical Review E 90, 042301 (2014).

[40] T. Bryk, A. Huerta, V. Hordiichuk, and A. D. Trokhymchuk, The Journal of Chemical Physics 147, 064509 (2017)

[41] K. Trachenko and V. V. Brazhkin, Reports on Progress in Physics 79, 016502 (2015).

[42] L. Wang, C. Yang, M. T. Dove, Y. D. Fomin, V. V. Brazhkin, and K. Trachenko, Physical Review E 95, 032116 (2017)

[43] N. P. Kryuchkov, V. V. Brazhkin, and S. O. Yurchenko, The Journal of Physical Chemistry Letters 10, 4470 (2019)

[44] A. E. Sherwood and J. M. Prausnitz, The Journal of Chemical Physics 41, 429 (1964)

[45] R. G. Gordon and Y. S. Kim, The Journal of Chemical Physics 56, 3122 (1972).

[46] R. Ramírez and C. P. Herrero, The Journal of Chemical 
Physics 129, 204502 (2008).

[47] W. G. Hoover, D. A. Young, and R. Grover, The Journal of Chemical Physics 56, 2207 (1972).

[48] S. Prestipino, F. Saija, and P. V. Giaquinta, The Journal of Chemical Physics 123, 144110 (2005).

[49] A. B. Belonoshko, R. Ahuja, and B. Johansson, Physical Review Letters 84, 3638 (2000)

[50] J. G. Williams, A. S. Konopliv, D. H. Boggs, R. S. Park, D.-N. Yuan, F. G. Lemoine, S. Goossens, E. Mazarico, F. Nimmo, R. C. Weber, S. W. Asmar, H. J. Melosh, G. A. Neumann, R. J. Phillips, D. E. Smith, S. C. Solomon, M. M. Watkins, M. A. Wieczorek, J. C. Andrews-Hanna, J. W. Head, W. S. Kiefer, I. Matsuyama, P. J. McGovern, G. J. Taylor, and M. T. Zuber, Journal of Geophysical Research: Planets 119, 1546 (2014)

[51] D. L. Anderson, Theory of the Earth (Boston, Blackwell Publications, 1989).

[52] See Supplementary Materials (URL).

[53] S. Plimpton, Journal of Computational Physics 117, 1
(1995)

[54] S. A. Khrapak, N. P. Kryuchkov, and S. O. Yurchenko, Physical Review E 97, 022616 (2018)

[55] S. O. Yurchenko, K. A. Komarov, N. P. Kryuchkov, K. I. Zaytsev, and V. V. Brazhkin, The Journal of Chemical Physics 148, 134508 (2018).

[56] S. O. Yurchenko, N. P. Kryuchkov, and A. V. Ivlev, The Journal of Chemical Physics 143, 034506 (2015).

[57] Y. Amo and Y. Tominaga, Physical Review E 60, 1708 (1999)

[58] G. E. Walrafen, Y. C. Chu, and G. J. Piermarini, J. Phys. Chem. 100, 10363 (1996).

[59] C. H. Wang and R. B. Wright, J. Phys. Chem. 55, 1617 (1971)

[60] F. Demmel, A. Fraile, D. Szubrin, W.-C. Pilgrim, and C. Morkel, Journal of Physics: Condensed Matter 27, $455102(2015)$

[61] F. Demmel and A. Tani, Physical Review E 97, 062124 (2018)

[62] F. Demmel, Physical Review B 101, 014207 (2020) 